\title{
Changes in dominant gut microbial species and metabolites in children with Crohn's disease during exclusive enteral nutrition
}

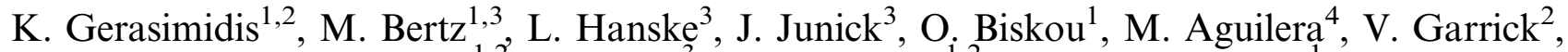 \\ R. K. Russell ${ }^{1,2}$, M. Blaut ${ }^{3}$, P. McGrogan ${ }^{1,2}$ and C. A. Edwards ${ }^{1}$ \\ ${ }^{1}$ Human Nutrition, College of Medicine, Veterinary and Life Sciences, University of Glasgow, Glasgow, G3 8SJ, \\ ${ }^{2}$ Department of Pediatric Gastroenterology, Hepatology and Nutrition, Royal Hospital for Sick Children, Glasgow, \\ G3 8SJ, ${ }^{3}$ Department of Gastrointestinal Microbiology, German Institute of Human Nutrition Potsdam-Rehbruecke, \\ Germany and ${ }^{4}$ Department of Microbiology, Faculty of Pharmacy, University of Granada, Granada, Spain
}

The gut microbiota is implicated in the pathogenesis of Crohn's Disease (CD) ${ }^{(1)}$. Exclusive enteral nutrition (EEN) is a successful treatment but its mode of action remains unknown ${ }^{(2)}$. We assessed changes in the fecal microbiota milieu during EEN.

Five faecal samples were collected from CD children; 4 during EEN (start, 15, 30, end EEN 60 days) and a fifth on free habitual diet. Two samples were collected from healthy controls. Faecal pH, bacterial metabolites (Figure) and quantitative changes (q-PCR) of total and 7 major bacterial groups implicated in CD were measured.

68 samples were from $15 \mathrm{CD}$ children and 40 from 21 controls. Faecal pH and total sulphide increased and butyric acid decreased during EEN (Figure). F. prausnitzii spp. concentration significantly decreased after $30 \mathrm{~d}$ on EEN (Figure). In patients who responded to EEN, the magnitude of the observed changes was greater and the concentration of Bacteroides/Prevotella group also decreased. All these changes reverted to pre-treatment levels when the children returned to their free habitual diet.
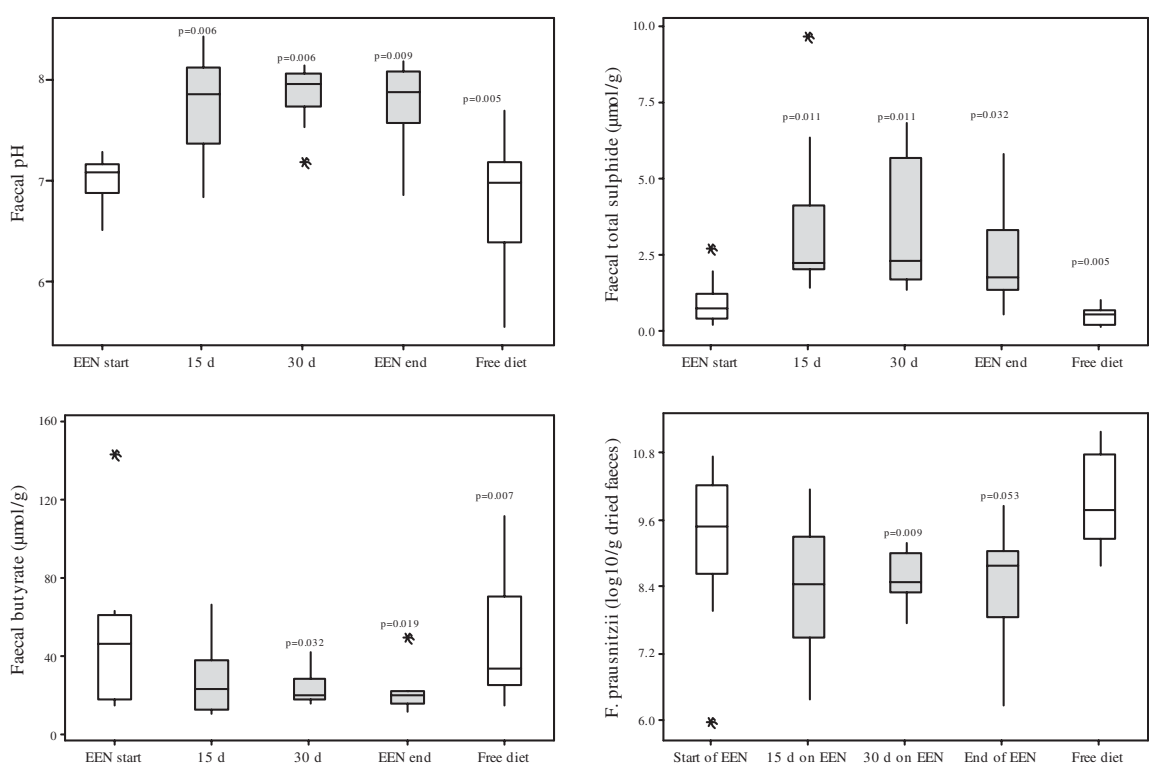

Fig. 1. Fecal $\mathrm{pH}$, and concentrations (g/dry feces) of total sulphide, butyrate and F. prausnitzii spp. before, during and after EEN completion (free habitual diet)

EEN impacts on gut microbiota composition and changes faecal metabolic activity. It is difficult to infer a causative association between such changes and disease improvement but the results do challenge the current perception of a protective role for F. prausnitzii in $\mathrm{CD}$.

KG was funded by the Greek State Scholarship Foundation, the Hellenic Foundation of Gastroenterology \& Nutrition, the Barr Endowment Fund and the Yorkhill Children Charity.

1. Sartor RB (2008) Gastroenterology 134, 577-94.

2. Gerasimidis K et al. (2011) J Clin Gastroenterol 45, 234-9. 